\title{
Toll like receptor 2(TLR2) in acne vulgaris patients before and after oral isotretinion therapy
}

*Wakas Saadi Mahmood, ** Nameer Kareem Qasim, *** Nahla Ghanim Abdulmajeed

\section{ABSTRACT}

Background: Acne vulgaris is one of the top three most commonly encountered dermatological problems worldwide in both primary and secondary care. Human keratinocytes express functional TLR2 heterodimers. An increased expression of TLR2 was detected in the epidermis of inflammatory acne lesions, as observed in normal skin; the expression level increased with the degree of differentiation of the keratinocytes. TLR2 expression is upregulated in inflammatory acne lesions and induced by C. acnes. The current study conducted to assess the oral isotretinion treatment effect on the acne vulgaris patients by evaluated the Toll Like Receptor 2 as a major immune system marker in Acne vulgaris immune response.

Methods: 50 patients with acne vulgaris seen in outpatient clinic of dermatology centre(AlBaidhaa out clinic center) in Baghdad during the period $1 / 9 / 2017$ to $1 / 8 / 2018$. The diagnosis of patients were done by the specialist dermatologist only sever and moderate acne cases were chosen. Fifty serum samples were collected before oral isotretinion therapy, only forty-five serum samples were collected after 8 weeks of oral isotretinion therapy. All patients have received $0.5 \mathrm{mg}$ $1 \mathrm{mg} / \mathrm{kg} /$ day of oral isotretinoin. The mean ages was 22 years(range, 16-36 years). A total of 50 patients, $36(72 \%)$ of patients were females and 14(28\%) were males. Furthermore $43(86 \%)$ of cases have heredity history. Also control serum samples were collected from people without acne and they chose with the same mean ages. The serum samples were stored at $-70^{\circ} \mathrm{C}$ for up to six months. ELISA test for quantitative determination of TLR2 human was done. Statistical analysis was done using T-test.

Results: Highly significant statistical difference were noticed $(\mathrm{p}<0.001)$ when we compare study group after therapy with control group, also highly significant statistical difference was noticed $(p<0.01)$ when we compare study group before therapy with control group.

Conclusion: Significant decrease of TLR-2 level was noticed in study group after isotretinoin therapy in comparison with same group before therapy and also with the control group. Pronouncedly the effects of oral isotretinion suggest a path towards importance of this therapy as an anti-acne agent

Key words: Toll Like Receptor 2(TLR2), Cutibacterium acnes, Acne vulgaris, Oral Isotretinion.

Corresponding address to: Biology department/Science collage/Tikrit university; Medical collage/ Ibn-Sina university/of medical and pharmaceutical sciences; Immunological laboratory Center/ Medical city

Received at $15 / 2 / 2019$

Accepted at 3/5/2019

\section{INTRODUCTION}

Acne vulgaris affecting more than $85 \%$ of adolescents, and can also persist into adulthood ${ }^{(1)}$ Acne vulgaris is the most common chronic diseases of pilosebaceous unit characterized by non-inflammatory lesions of open and closed comedons, and inflammatory lesions of papules, pustules and nodules (2). The pathology of this condition is complicated. The pathogenesis of acne is multifactorial, including seborrhea, hyperkeratinization, hypercolonization of Cutibacterium acnes, and inflammation ${ }^{(3)}$.

Acne pathophysiology are, inflammatory events mediated by interleukins, $C$. acnes activates the innate immune system via Toll-like receptors
(TLRs), and sebaceous gland lipids influence the innate immune system (4). Human keratinocytes express functional TLR2 heterodimers. An increased expression of TLR2 was detected in the epidermis of inflammatory acne lesions, as observed in normal skin; the expression level increased with the degree of differentiation of the keratinocytes. TLR2 expression is upregulated in inflammatory acne lesions and induced by $C$. acnes. In inflammatory acne lesions, increased epidermal TLR2 expression facilitates recognition of $C$. acnes and contributes to inflammatory responses. Keratinocytes are one of the major players in the pathophysiology of acne, in which TLR2 
recognition and activation can be the initiating step in comedogenesis ${ }^{(12,5)}$.

There is no ideal treatment for acne, although a suitable regimen for reducing lesions can be found for most patients. The aims of treatment of acne vulgaris are to prevent long -term complications. Oral isotretinoin is the most effective therapy in Acne vulgaris ${ }^{(6)}$.

The aim of current study is to assess the quality of oral isotretinion as a major treatment of acne vulgaris, which can inhibit the level of TLR2, which will provide promising benefits to patients with acne vulgaris.

\section{METHODS}

The work was done in the laboratories of Department of Immunology laboratory/ Health center laboratories/Medical city, where these devices, tools and materials are available, whether glasses or chemicals in these laboratories.

This study is a prospective study one and conducted during the period September 2017 to August 2018. Fifty patients with acne were recruited of dermatology clinics (Al-Baidhaa out clinic center/Baghdad city). The diagnosis of acne was made by specialist dermatologists. All patients have received $0.5 \mathrm{mg}-1 \mathrm{mg} / \mathrm{kg} /$ day of oral isotretinoin (7). All patients have been serum sampled before treatment, and $45(90 \%)$ of patients were sampled after 8 weeks of therapy whereas other cases was default. Also control serum samples were collected from people without acne and they chose with the same mean ages. Whole blood specimens were collect using acceptable medical techniques to avoid hemolysis. Blood was allowed to clot and the serum was separated by centrifugation. Then 2cc from each sample were transported from plain tube into $2 \mathrm{cc}$ eppendorf's tube. Specimens were stored at $-70^{\circ} \mathrm{C}$ for up to six months ${ }^{(8)}$ ELISA test for quantitative determination of TLR2 human was done. This test was performed following the procedure protocol included within the kit packing as issued from the manufacturer company (CUSABIO, Germany).

This assay employs the quantitative sandwich enzyme immunoassay technique. Antibody specific for TLR2 has been pre-coated onto a microplate. Standards and samples are pipetted into the wells and any TLR2 present is bound by the immobilized antibody. After removing any bound substances, a biotinconjugated antibody specific for TLR2 is added to the wells. After washing, avidin conjugated Horseradish Peroxidase(HRP) is added to the wells. Following a wash to remove any unbound avidin-enzyme reagent, a substrate solution is added to the wells and color develops in proportion to the amount of TLR2 bound in the initial step. The color development is stopped and the intensity of the color is measured.

Preparation of reagents: Biotinantobody(1x): Mix 10 $\mu 1$ of Biotinantibody+ $990 \mu 1$ of Biotin-antibody Diluent. HRP-avidin(1x): Mix 10 $\mu$ l of HRP-avidin+ $990 \mu 1$ of HRP-avidin Diluent. Wash Buffer(1x): Dilute $20 \mathrm{ml}$ of wash buffer concentrate $(25 \mathrm{x})$ into deionized or distilled water to prepare $500 \mathrm{ml}$ of wash buffer (1x). Standard: Reconstitute the standard with $1.0 \mathrm{ml}$ of sample diluents. Do not substitute other diluents. Gently agitation for 15 minutes. Then pipette $250 \mu$ l of sample diluent into each tube.

Test Protocol: Prepare reagents, sample and standards as instructed. Add $100 \mu \mathrm{l}$ standard or sample to each well. Incubate 2 hours at $37^{\circ} \mathrm{C}$. Remove the liquid of each well, do not wash. Add $100 \mu \mathrm{l}$ Biotin-antibody $(1 \mathrm{x})$ to each well. Incubate 1 hour at $37^{\circ} \mathrm{C}$. Aspirate and wash 3 times. Add $100 \mu \mathrm{l} \mathrm{HRP-avidin(1x)}$ to each well. Incubate 1 hour at $37^{\circ} \mathrm{C}$. Aspirate and wash 5 times. Add $90 \mu \mathrm{l}$ TMB Substrate to each well. Incubate 15-30 minutes at $37^{\circ} \mathrm{C}$ (protect from light). Add $50 \mu 1$ stop solution to each well. Read at $450 \mathrm{~nm}$ within 5 minutes.

Statistical analysis was done using statistical package for social sciences version 22 (SPSS V.22, Chicago, IL, USA). Two samples unpaired and paired T-test were used to find the means of normally continuous samples of two set of data. All $\mathrm{P}$ values used were asymptotic and two sided. Findings with $P$ value less than 0.05 were considered significant. 


\section{RESULTS and DISCUSSION}

Many patients admitted into the outpatient clinic of dermatological care center in Baghdad city with acne vulgaris. Only 50 patient were recruited in this study depending on the clinical diagnosis and investigations. After sample collection, all patients will received oral isotretinoin for 8 weeks. After this period only 45 patients returns into the clinic centre and samples have been collected again. From 50 cases of acne vulgaris, 36 $(72 \%)$ of patients were females, while $14(28 \%)$ were males with female to male ratio of about (4:1). Also, The mean ages was $22 \pm 16-36$ years. The number of teens

Table (1): Statistical comparison between study group before and after therapy with control group in regard to TLR-2.

\begin{tabular}{|l|c|c|c|c|c|}
\hline \multicolumn{1}{|c|}{ Categories } & Mean U/ml & SD $*$ & T- test & Df $* *$ & P-value \\
\hline TLR-2 before therapy $(\mathrm{n}=45)$ & 41.669 & 84.518 & 3.072 & 83 & 0.003 \\
\cline { 1 - 3 } Control group $(\mathrm{n}=40)$ & 0.583 & 1.258 & & & \\
\cline { 1 - 3 } TLR-2 after therapy $(\mathrm{n}=45)$ & 9.838 & 7.086 & 8.139 & 83 & $<0.001$ \\
\cline { 1 - 2 } Control group $(\mathrm{n}=40)$ & 0.583 & 1.258 & & & \\
\hline
\end{tabular}

$* \mathrm{SD}=$ Standard deviation, ** $\mathrm{Df}=$ Degree of freedom

To compare both means of study group before and after therapy, paired T- test was used with significant statistical difference $(\mathrm{P}<0.05)$ as shown in table $(2)$.

Table (2): Statistical comparison between study group before and after therapy in regard to TLR-2.

\begin{tabular}{|l|c|c|c|c|c|}
\hline \multicolumn{1}{|c|}{ Categories } & Mean U/ml & SD $*$ & T- test & Df ** & P- value \\
\cline { 1 - 3 } TLR-2 before therapy $(\mathrm{n}=45)$ & 41.669 & 84.518 & 2.517 & 88 & 0.014 \\
\cline { 1 - 3 } TLR-2 after therapy $(\mathrm{n}=45)$ & 9.838 & 7.086 & & & \\
\hline
\end{tabular}

$* \mathrm{SD}=$ Standard deviation, $* * \mathrm{Df}=$ Degree of freedom

The TLRs are the main marker of innate immunity ${ }^{(9)}$. The ability of $C$. acnes to activate TLR-2 might be explained by the distinct composition of peptidoglycan in their cell wall compared to other Grampositive bacteria. Activation leads to the release of proinflammatory cytokines (interleukin-1 $\alpha,-8$, and -12) and tumor necrosis factor alpha (TNF- $\alpha$ ) by immune cells (keratinocytes and monocytes), thereby modulating the host immune response ${ }^{(10) \text {. }}$

The TLR2 is expressed on the cell surface of macrophages surrounding pilosebaceous follicle in acne lesions ${ }^{(11)}$. Inflammation triggered through the TLR2 is important in the pathogenesis of Acne vulgaris. TLR2 expression in vivo is increased in epidermis of acne lesions ${ }^{(5)}$. The C.acnes-derived GroEL, DnaK, or lipoglycans may acts as ligands for the TLRs. Also, the membrane fraction of $C$. acnes containing peptidoglycan and lipoteichoic acid resulted in an increase of keratinocyte proliferation. This suggests that $C$. acnes acts in the initial phase of acne ${ }^{(12) \text {. }}$

Systemic retinoids modulate TLRs expressed by kerationcytes or monocytes ${ }^{(9)}$. Dispenza et al. reported that this was due to a significant decrease in TLR-2 expression by monocytes, with a subsequently lower cytokine response ${ }^{(13) .}$ Increasing evidence shows that $C$. acnes activates toll-like receptor 2 (TLR2) to induce various proinflammatory cytokines (14). By using cell culture models and immunohistochemistry, Kim et al. showed that $C$. acnes triggers an inflammatory cytokine response in macrophages by the activation of TLR-2 ${ }^{(10) \text {. }}$

Furthermore, $C$. acnes colony in pilosebaceous unit disrupt the epithelium of follicle, from where the bacterial cell leaks out and get into contact with 

myeloid cells (like macrophage) via TLR2
$(15,16)$.

Current results showed a highly significant increase of TLR-2 in study group of acne patients before and after therapy, with significance ulteration (i.e. down- regulation) of these receptors after isotretinoin treatment.

\section{CONCLUSION}

Significant decrease of TLR-2 level was noticed in study group after isotretinoin therapy in comparison with same group before therapy and also with the control group. That reveal the importance of oral isotretinoin therapy in prevent the interactions between immune system and C.acnes by decreasing the levels of TLR2. Pronouncedly the effects of oral isotretinion suggest a path towards importance of this therapy as an anti-acne agent. The increasing knowledge of TLR and it is effect on innate immunity are modifying our concepts about comedogenesis and inflammation that result in clinical acne lesions.

\section{REFRENCES}

1. Williams, C.H., Delavalle, P.R., \& Garner, S.(2012). Acne Vulgaris. The lancet. 379:361-72.

2. Webster, G.F., Leyden, J.J., Tsai, C.C., Baehni, P. \& McArthur, W.P. (1980). Polymorphonuclear leukocyte lysosomal release in response to Propionibacterium acnes in vitro and it is enhancement by sera from inflammatory acne patients. Journal of Investigate Dermatology. 74(6): 398-401.

3. Shaleen, B., \& Gonzalez, M.(2011). A microbial etiology of acne: what is the evidence. British. Journal of Dermatology. 165: 474-485.

4. Shalita, A.R., DelRosso, J.Q. \& Webster, G.F.(2011). Acne Vulgaris. Informa Health Care. American Acne and Rosacea Society.

5. Su, Q., Grabowski, M. \& Weindi, G.(2016). Recognition of Propionibacterium acnes by human TLR2 heterodimer. International Journal of Medical Microbiology.

6. Mahmood, N.F., \& Shipman, R.A.(2017). The age-old problem of acne. International Journal of Women's Dermatology. ELSEVIER. 3:71-76.

7. Coats, P., Adam, C.A., Cunliffe, W.J., McGinley, K.T., Early, A.E., Leyden, J.J.,
Ravenscroft, J., Vyakrnam, S. \& Vowels, B.(1997). Does oral isotretinoin prevent Propionibacterium acnes resistance? KARGER. 195:4-9.

8. Issa, S.B.(2013). The role of AntiLysozyme and Anti-Lactoferrin Autoantibodies in chronic inflammatory bowel diseases: serological study. Thesis submitted for Iraqi board for medical spacializations in pathology. 29.

9. Beylot, C., Auffret, N., Poli, F., Claudel, J.P., Leccia, M.T., DelGiudice, P., \& Dreno,B.(2014). Propionibacterium acnes: an update on it is role in the pathogenesis of acne. JEADV. 28:271-278.

10. Achermann, Y., Goldstelin, C.T., Coenye, T. \& Shirttiff, E.M.(2014). Propionibacterium acnes: from commensal to opportunistic Biofilm associated Implant pathogen. CMR. Journals ASM. 27(3):419440.

11. Omar, H., McDowell, A. \& Alexeyev, A.O.(2016). Understanding the role of Propionibacterium acnes in acne vulgaris: the critical importance of skin sampling methodologies. Clinics in Dermatology.

12. Dessinioti, C., Anddreas, D., \& Katsamba, M.(2010). The role of Propionibacterium acnes in acne pathogenesis: Facts and controversies. Clinics in Dermatology. Elsevier. 28:2-7.

13. Dreno, B., Gollnick, H.; Kang, S., Thiboutot, D., Bettoli, V., Torres, V., \& Leyden, J.(2015). Understanding innate immunity and inflammation in acne: implications for manangement. Journal of European Academy of Dermatology and Venereology. 29:3-11.

14. Xia, X., Li, Z., Liu, K., Wu, Y., Jiang, D. \& Lai, Y.(2016). Staphylococcal LTA-induced miR-143 inhibits Propionibacterium acnesmediated inflammatory response in skin. Journal of Investigate Dermatology. 136: 621-630.

15. Qidwai, A., Pandey, M., Pathak, S., Kumar, R., \& Dikshit, A.(2017). The emerging principles for acne biogenesis: A dermatological problem of puberty. Journal of Human Microbiome. ELSEVIER. 24522317.

16. Kwon, H.H., Yoon, J.Y., Park, S.Y. \& Suh, D.H.(2013). Analysis of distribution patterns of Propionibacterium acnes phylotypes and peptostreptococcus species from acne lesions. British Journal of Dermatology. 169: 11521155. 